\title{
Variation in Spontaneous Activity and Visual Evoked Response in Primary Visual Cortex of the S334ter-3 Rats
}

\author{
Bojun Hou ${ }^{1}, \mathrm{Ke} \mathrm{Chen}^{1 *}$, Yilei Zhao ${ }^{1}$, Leanne Lai Hang Chan ${ }^{2,3^{*}}$ \\ ${ }^{1}$ The Clinical Hospital of Chengdu Brain Science Institute, MOE Key Lab for NeuroInformation, University of Electronic Science and \\ Technology of China, Chengdu 610054, People's Republic of China \\ ${ }^{2}$ Department of Electrical Engineering, City University of Hong Kong, Tat Chee Avenue, Hong Kong SAR, China \\ ${ }^{3}$ Centre for Biosystems, Neuroscience, and Nanotechnology, City University of Hong Kong, Tat Chee Avenue, Hong Kong SAR, China
}

\begin{abstract}
S334ter-3 retinal degeneration (RD) rats have been widely used to investigate degenerative diseases of the retina. In this model, morphological and electrophysiological changes have been observed in the retina, superior colliculus and primary visual cortex (V1). In this study, experimental rats (S334ter-3) carried one copy of the mutant transgene. We measured the extracellular responses in the primary visual cortex to three stimulus contrast levels (spontaneous activity, medium contrast, and high contrast) at the preferred parameters of each recorded cell under classical receptive field (CRF) stimulation. Then we compared the responses (spontaneous activity and the visual evoked responses) in RD rats with those in wildtype rats. Our results show that V1 cells in the RD group exhibit stronger spontaneous activity but weaker stimulus-evoked responses at medium and high contrasts. At the same time, compared with WT group, $\mathrm{RD}$ group also showed a narrow dynamic range. These results indicate the decrease in discriminating the stimuli contrast and loss in responses and lower signal to noise ratio after retina degeneration.
\end{abstract}

\section{INTRODUCTION}

The retina consists of a sophisticated neural circuitry that decomposes the retinal image into signals representing contrast and movement. These representations are then transmitted through the optic nerve and lateral geniculate nucleus to the primary visual cortex (V1), which uses them to analyze shapes and other features of visual objects. In the mammalian V1, striate neurons are exquisitely tuned to respond to specific spatio-temporal variations in luminance contrast.

Mammalian retinal degeneration initiated by gene defects in rods, cones or the retinal pigmented epithelium often triggers loss of photoreceptors in the retina, effectively leaving the neural retina deafferented. Many previous studies have found a significant increase in spontaneous activity throughout the visual system in response to retinal degeneration in various animal models [1-8]. Humans with Retinitis Pigmentosa (RP), the most common form of retinal degeneration, and more than $25 \%$ of human RP cases are caused by the rhodopsin gene mutation [9]. The S334ter-3 rat is a transgenic model of retinal degeneration developed to express a rhodopsin mutation similar to the RP that caused by rhodopsin mutation. That model express rhodopsin gene with an early termination codon at residue 334, resulting in the expression of a rhodopsin protein without $15 \mathrm{C}$-terminal amino acids that are involved in rhodopsin trafficking to the photoreceptor outer segments and in the inactivation of rhodopsin protein after light absorption. That model usually uses heterozygous rats instead of homozygote in order to avoid any changes in the retina attributable to albinism (homozygous S334ter-3 rats are albino). In a previous morphological study [9], a progressive decrease in the thickness of the outer nuclear layer (ONL) was observed in heterozygous S334ter-3 rats with the absence of photoreceptor layers both in the central and peripheral retinas. And indicates that RD rats of S334ter-3 model at P60 are at a stage of retinal degeneration that has similar characteristics to the middle stage of degeneration in human RP patients: cone photoreceptors are somewhat affected but rods are significantly withered, with few remaining.

Previous studies investigated the degeneration of visual electrophysiology in various types of degeneration models [10-16]. What remains unclear is how the responses of V1 neurons to variations in luminance contrast change in these models of RP. The present study investigates this question.

\section{Materials and Methods}

\subsection{Animal Preparation and Maintenance}

All animals were obtained from the Laboratory Animal Services Centre of Chinese University of Hong Kong and housed in animal facilities at City University of Hong Kong. Ten Long-Evans and 11 S334ter-3 rats (3-4 
months of age, 55-70 days) were used as the wildtype (WT) and RD models, respectively. S334ter-line-3 rat litters were obtained by breeding transgenic homozygotes (with two copies of the mutant transgene) with LE rats. Hence, the experimental rats (S334ter-3) carried one copy of the mutant transgene.

Acute recordings were conducted in $\mathrm{WT}$ and $\mathrm{RD}$ rats. Initially, animals were anesthetized by intraperitoneal injection of a ketamine-xylazine combination (ketamine: $70 \mathrm{mg} / \mathrm{kg}$ and xylazine: $7 \mathrm{mg} / \mathrm{kg}$; Alfasan International B.V., Holland); anesthesia was maintained at $2 \%$ isoflurane (RWD Life Science, Shenzhen, China) during recordings. After a craniotomy (A-P: Lambda $-0.51 \mathrm{~mm}$ to Lambda $+1.67 \mathrm{~mm}$; M-L: $-2.5 \mathrm{~mm}$ to $-3.0 \mathrm{~mm}$; D-V: 0 to $1 \mathrm{~mm}$ ) over V1, a bone screw was fixed to the skull (as an electrical ground) and a tungsten electrode was placed on the surface of V1.

\subsection{Visual Stimulation and Recording}

An A-M Systems 3600 amplifier (A-M Systems, USA) and a data acquisition card (National Instrument, USA) were used for recording neural responses and spike sorting. Tungsten-in-glass microelectrodes were used to record single units extracellularly. Single-unit responses were bandpass filtered at $0.5-5 \mathrm{kHz}$ and then sampled at $40 \mathrm{kHz}$.

Various stimuli were displayed across the animal's visual field to activate V1. We recorded units at several cortical depths: after completing the stimulus set at one depth, the microelectrode was advanced slowly by an oil hydraulic micromanipulator (MO-10, Narishige, Japan) and stimulus presentation was resumed.

Stimuli were presented on a Dell monitor (screen size: $35 \times 26 \mathrm{~cm}$, frame rate: $60 \mathrm{~Hz}$, and resolution: $800 \times 600$ pixels) positioned $23 \mathrm{~cm}$ from the rat's eye (the screen occupied $75 \times 58^{\circ}$ in the visual field). The mean luminance was $26 \mathrm{~cd} / \mathrm{m} 2$. All measurements were made during the stimulation of the rat's contralateral eye and masking the ipsilateral eye by insulated rubber tape (Fig. 1a). At each cortical depth we determined the responding cells' preferred stimulus parameters (spatial frequency: 0.04-0.16 cpd; temporal frequency: 1-4 Hz) under classical CRF stimulation (Fig. 1b). The CRF profile and center location were carefully determined by placing a narrow sine-wave grating patch at successive positions along the horizontal and perpendicular axes.

Finally, by using the resulting preferred parameters, we measured the cells' responses to three different stimulus contrasts levels: spontaneous activity ( $0 \%$ contrast), responses at medium contrast (30\% contrast) and to high contrast $(100 \%$ contrast $)$ stimuli. Each measurement was presented for 5-10 cycles of the grating drift, and standard errors were calculated for 3-10 repeats. Peri-stimulus time histograms (PSTHs) of unit responses were generated and analyzed on-line using custom-made software.

All experimental procedures were approved by the Animal Subjects Ethics Sub-Committees of City University of Hong Kong and the Health Department of Hong Kong Special Administrative Region. All methods were carried out in accordance with the approved welfare guidelines of the Animal Subjects Ethics Sub-Committees of City University of Hong Kong and the Health Department of Hong Kong Special Administrative Region. (a)

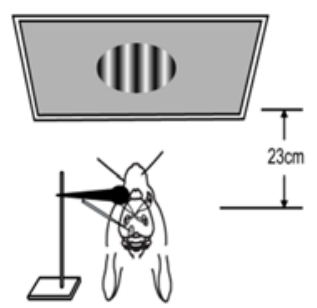

(b)

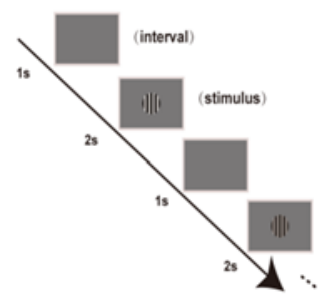

Fig. 1. Schematic diagram of experimental measurement. (a) The response of the rat's contralateral eye was measured while the ipsilateral eye was masked with an insulated rubber tape. (b) The responses of the cells to three different stimulus contrasts levels were measured using CRF stimulation with preferred parameters. Each grating patch was presented for 2 seconds with an interval of 1 second.

\subsection{Data Analysis}

Neuronal activity is reported either as frequency (spikes/s) or normalized activity (z-score transformation). All population values given below are expressed as the mean plus or minus the standard deviation. And all two-way comparisons were tested for significance with the MannWhitney U test.

\section{Results}

To characterize the quantitative relationship of neural response and contrast, we recorded neural responses (by using sinusoidal gratings at the optimal orientation, spatial frequency and temporal frequency under CRF stimulation) of each cell in the RD and WT group at three contrast levels: spontaneous activity ( $0 \%$ contrast), responses at medium contrast (30\% contrast) and to high contrast $(100 \%$ contrast $)$ stimuli. Similar to previous reports [6], the RD group exhibited stronger spontaneous activity but a weaker stimulus-evoked responses. In addition, we can found that the response range of $\mathrm{RD}$ group was smaller. Figure 2 shows the average response of V1 neurons at $0 \%, 30 \%$ and $100 \%$ contrast in the RD and WT groups. The spontaneous activity was $4.4 \pm 3.7$ spikes/s (mean $\pm \mathrm{SD}$; range: 0 to 13.44 spikes/s) in the WT group and $6.7 \pm 7.8$ spikes $/ \mathrm{s}$ (mean $\pm \mathrm{SD}$; range: 0.3 to 33.1 spikes/s) in the RD group. Under medium contrast stimulation $(30 \%)$ the firing rates were $9.7 \pm 7.8$ spikes/s in the WT group and $8.5 \pm 7.9$ spikes/s in the RD group. Finally, at high contrast $(100 \%)$ the firing rates were 13.9 $\pm 9.6 \mathrm{spikes} / \mathrm{s}$ in the WT group and $10.7 \pm 7.6 \mathrm{spikes} / \mathrm{s}$ in the $\mathrm{RD}$ group. These differences in firing rate in spontaneous, medium-level contrast, and high contrast between the WT and RD group were significant $(\mathrm{p}<0.001$ for all comparisons). 
(a)

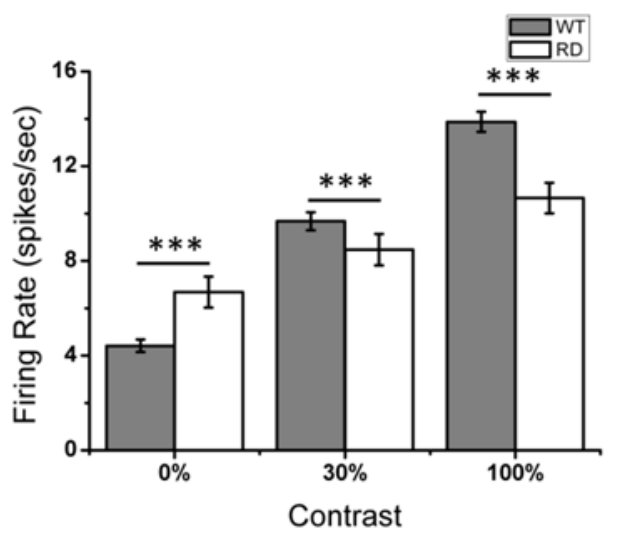

Fig. 2. Comparison of V1 neuronal responses under $0 \%$, 30\% and $100 \%$ contrast between the LE and RD rats. Average firing rate between the $\mathrm{WT}$ and $\mathrm{RD}$ groups under different contrast conditions. Error bars represent the SEM values. "***" indicates $\mathrm{P}<0.001$ in the comparison.

\section{Discussion}

Though in S334ter-3 rats the pathological changes occur in the retina itself, the concurrent reorganization in the primary visual cortex may be an important factor in the perceptual effects of retinal degeneration. The S334ter-3 model rats used in this study exhibited a stronger spontaneous activity but weaker visual evoked responses compared to WT rats. This result is consistent with previous studies in the retina, superior colliculus and primary visual cortex of different mouse and rat RD model [1-6]. Dräger and Hubel also found that spontaneous activity in V1 disappeared when the corresponding eye was asphyxiated, suggesting that spontaneous activity is spurious and arises in the retina [17]. However, only minor morphological changes have been shown to occur in ganglion cells during RD [18]. These findings suggest that the emergence of spontaneous activity in the degenerating retina does not depend on gross anatomical rewiring of the retinal circuitry. Moreover, many studies have found that spontaneous activity arises at the level of the AII)-amacrine/ON cone bipolar cell network [19]. The primary source of this spontaneous noise lies in the electrically coupled network of AII-amacrine and ON cone bipolar cells. This coupled network appears to become spontaneously active partly due to a small hyperpolarization that occurs after loss of photoreceptor input during RD.

In our study, the spontaneous neural response was larger in the RD rats than in WT rats, but the visual evoked neural responses were weaker. This result advance our understanding of the functional changes in contrast detection associated with retinal degeneration, and indicates that while vision in $\mathrm{RD}$ rats is partially impaired at the middle stage of degeneration, the remaining neurons in the visual pathway are still able to capture some information about the stimuli, generating a weaker stimulus-evoked response in V1 compared in the control group.

\section{Acknowledgments}

This study was supported by the National Natural Science Foundation of China (Grant No. 81861128001, No. 61773094, No. 81400401), the 111 project B12027, and the Project of Science and Technology Department of Sichuan Province (2017HH0001), Research Grants Council of the Hong Kong Special Administrative Region, China (grant No. CityU 11208218), and the City University of Hong Kong (grant No. CityU 7004831).

\section{References}

1. M. Pu, L. Xu, H. Zhang, Ophthalmol. Vis. Sci. 47, 3579-3585 (2006)

2. D.J. Margolis, G. Newkirk, T. Euler, P.B. Detwiler, J. Neurosci. 28, 6526-6536 (2008)

3. J. Borowska, S. Trenholm, G.B. Awatramani, J. Neurosci. 31, 5000-5012 (2011)

4. Y.S. Goo, K.N. Ahn, Y.J. Song, S.H.Ahn, S.K. Han, S.B. Ryu, K.H. Kim, Korean J. Physiol. Pharmacol 15, 415-422 (2011)

5. C. Sekirnjak, L.H. Jepson, P. Hottowy, A. Sher, W. Dabrowski, A.M. Litke, E. Chichilnisky, J. Neurophysiol 105, 2560-2571 (2011)

6. Y. Wang, K. Chen, P. Xu, T.K. Ng, L.L.H. Chan, Neurosci. Lett. 623, 42-46 (2016)

7. T.J. Mcgill, G.T. Prusky, R.M. Douglas, et al., Ophthalmol. Vis. Sci. 53, 6232-6244 (2012)

8. Y. Wang, K. Chen, L.L. Chan, Neuroscience 383, 84-97 (2018)

9. A. Ray, et al., Cell Tissue Res. 339, 481-491 (2010)

10. C. Sekirnjak, C. Hulse, L.H. Jepson, P. Hottowy, A. Sher, W. Dabrowski, A.M. Litke, E.J. Chichilnisky, J. Neurophysiol 102, 3260-3269 (2009)

11. C. Gias, A. Vugler, J. Lawrence, A.J. Carr, L.L. Chen, A. Ahmado, M. Semo, P.J. Coffey, Vision Res. 51, 2176-2185 (2011)

12. K. Chen, Y. Wang, X. Liang, Y. Zhang, T.K. Ng, L.L. Chan, Sci. Rep. 6, 26793 (2016)

13. M. M. LaVail, S. Nishikawa, R. H. Steinberg, et al., Exp. Eye Res. 167, 56-90 (2018)

14. N.Kiran, D. W. James, Trans. Vis. Sci. Tech. 7, 33 (2018)

15. A. Brun, X. Yu, C. Obringer, et al., Exp. Eye Res. 186, 107721 (2019)

16. B. Li, S. Gografe, A. Munchow, et al., Exp. Eye Res. 187, 107773 (2019)

17. E. Ivanova, C.W. Yee, B.R. Jr, B.T. Sagdullaev, Exp. Eye Res. 150, 81-89 (2016)

18. S. Trenholm, G.B. Awatramani, Front. Cell. Neurosci. 9, 277 (2015)

19. M.A. Sandberg, P.L. Sullivan, E.L. Berson, Investig. Ophthalmol. Vis. Sci. 21, 765-769 (1981) 